\title{
Mixtura social y sociabilidad: ¿̨inducen los Proyectos de Integración Social vínculos socioeconómicamente diversos?
}

Luis Vergara. Universidad de La Frontera, Temuco, Chile.

RESUMEN | La mixtura social se ha convertido en un objetivo de política pública en Chile y Latinoamérica. En principio, los barrios generados a partir de tal objetivo buscan disminuir la segregación de clase en la ciudad. Sin embargo, se desconoce si en las condiciones de mixtura y de entorno en que son construidos se puede (o no) promover sociabilidades pluriclasistas. Empleando dos proyectos de integración social y una metodología mixta, este artículo intenta llenar esa brecha. Los resultados sugieren que por el tipo de diversidad que promueven los barrios considerados, como también por su localización periférica en entornos homogéneos, las sociabilidades que producen no son lo suficientemente diversas, limitando el funcionamiento del llamado "efecto barrio". Los hallazgos revelan también la importancia de diseñar espacios de encuentro entre residentes, los que tienen capacidad de minimizar los habitus metropolitanos que despliegan las clases medias. Se concluye con sugerencias para mejorar y ampliar el enfoque de mixtura social.

PALABRAS CLAVE | política habitacional, integración social, segregación.

ABSTRACT | Social mixture has become an urban policy in Chile and Latin America. Although the corresponding initiatives seek to reduce the segregation of social classes, we still do not know whether, under the conditions of mixture and environment over which these neighbourhoods are built, it is possible (or not) to promote pluri-classist sociabilities. Using two social integration projects and a mixed methodology this article intends to fill this gap. The results suggest that due to the type of diversity promoted by these neighbourhoods, as well as because of their peripheral location in homogeneous environments, the sociabilities they produce are not diverse enough to work, for example, the so-called neighbourhood effect. The findings also reveal the importance of designing common spaces between residents, spaces that have the capacity to minimize metropolitan habitus that the middle classes deploy. It concludes with suggestions to improve and expand the social mix approach in housing policies.

KEYWORDS | housing policy, social integration, segregation.

Recibido el 7 de octubre de 2019, aprobado el 8 de diciembre de 2019

E-mail: luis.vergara@ufrontera.cl 


\section{Introducción}

La mixtura social se ha convertido en un objetivo de política urbana sobre el cual convergen las principales corrientes políticas chilenas, más allá de sus diferencias. Paulina Saball, ministra de Vivienda y Urbanismo durante el gobierno de Michelle Bachelet, declaró con posterioridad al encuentro Hábitat III de Quito en 2016 que "nuestro mayor desafío hoy es que los conjuntos habitacionales garanticen mixtura social”. Así también lo expresó Sebastián Piñera en su discurso con motivo de la entrega del proyecto de ley de Integración Social y Urbana en diciembre de 2018, el cual pretende crear Zonas de Integración Urbana, áreas donde habitarán familias de diferente condición socioeconómica a cambio de beneficios en las normas urbanísticas para las empresas inmobiliarias. Este acuerdo en materia de política urbana ha amortiguado el "pánico empresarial” hacia la mezcla social que -según Ruiz-Tagle y Romano (2019) - se dio cuando tales medidas comenzaron a aplicarse. Hoy, por el contrario, hay interés creciente en buena parte de las empresas inmobiliarias por desarrollar proyectos de mixtura, los que son percibidos como rentables (Sabatini, Mora, Polanco \& Brain, 2014), especialmente por el incremento económico que han experimentado los subsidios estatales asociados a ellos y -más ahora con la propuesta de Ley de Integración Social y Urbana- por los beneficios urbanísticos a los que podrán acceder.

Este trabajo se focaliza en un tipo específico de vecindarios de mixtura social: los Proyectos de Integración Social (PIS), que buscan reducir la segregación socioeconómica por medio de la proximidad espacial de familias de diferente nivel socioeconómico. En específico, se analiza cómo las condiciones de mixtura y de entorno sobre las cuales estos barrios son construidos influyen en la sociabilidad de sus residentes. Para ello, se despliega una aproximación socioespacial y multiescalar de la sociabilidad que se produce en estos vecindarios, argumentando que la proximidad no es suficiente para promover sociabilidades socioeconómicamente diversas. Se sostiene, en cambio, que es necesario tener en consideración el contexto social y espacial en el que se construyen estos barrios y que enfrentan, a lo largo de toda América Latina, estructuras urbanas segregadas como la de Santiago. A través del caso específico de Chile, se revelan algunas limitaciones que enfrenta una política habitacional que comienza a expandirse a lo largo de buena parte del continente (Vergara, 2019).

El artículo se compone de siete secciones. Primero se caracteriza la mixtura social chilena, mostrando las condiciones socioespaciales sobre las cuales se construyen los PIs. Luego se revisa cómo la sociabilidad es un fenómeno socioespacial y multiescalar, superando el énfasis sobre la proximidad con que operan los PIs. Enseguida se exponen los casos de estudio y la metodología empleada. A continuación se presentan los resultados: la sociabilidad intrabarrio; cómo la sociabilidad de quienes habitan en los PIs se extiende sobre la ciudad; y, finalmente, cómo el lugar en que se construyen estos barrios y las dotaciones que poseen actúan sobre la sociabilidad de los residentes. Se cierra con conclusiones que apuntan a mejorar la política de mixtura social, lo que es particularmente relevante en un contexto continental donde tal temática se expande dentro de la agenda urbana. 


\section{La mixtura social "a la chilena" y el énfasis en la proximidad}

La segregación socioeconómica se ha convertido en uno de los principales problemas urbanos en Chile, al igual que ocurre en buena parte de Latinoamérica. Este fenómeno, que sitúa a los pobres en los peores y más baratos lugares de la ciudad, ha generado serios problemas sociales, como procesos de guetificación en barrios de vivienda pública y desigualdad en las oportunidades de acceso a los servicios y equipamientos urbanos (Rodríguez \& Sugranyes, 2004). La segregación urbana en Chile no ha sido azarosa, sino el resultado de la combinación de varias medidas públicas que comenzaron a ser aplicadas a finales de los setenta y que se han proyectado sin grandes modificaciones hasta la actualidad; entre ellas, la liberalización del mercado de suelo (Sabatini, 2000); la construcción de barrios sobre la base de criterios de homogeneidad social; y la flexibilización de las normas constructivas y de localización de las viviendas públicas (Imilan, 2016). A esto se han sumado procesos de jibarización estatal y privatización que, desde la dictadura de Augusto Pinochet, han operado en materia de educación, salud, jubilación, movilidad, entre otros (Garretón, 1981). Se ha moldeado así una sociedad en que la clase alta no suele compartir espacios públicos de encuentro (escuelas, hospitales, plazas) con personas pobres (Comisión Económica para América Latina y el Caribe, Cepal, 2007; Stillerman, 2016). Los problemas de desigualdad han impulsado desde octubre de 2019 fuertes protestas sociales que han tenido su mayor expresión en las calles de Santiago.

A pesar de que diversos estudios advertían que la segregación y la desigualdad en Chile podían derivar en problemas de cohesión social (Rodríguez \& Sugranyes, 2004; Tironi, 2007), colocando en riesgo la base normativa que entregaba unidad a la sociedad (Rasse, 2015), pocas fueron las medidas que se diseñaron para frenarla. Una de las políticas pioneras en este ámbito ocurrió a nivel habitacional, con los llamados pIs. Hasta 2007, los barrios construidos con subsidios del Estado estaban dirigidos a grupos específicos de personas, generalmente de un mismo nivel socioeconómico. Había subsidios para familias pobres (Fondo Solidario de Vivienda, Fsv) y para familias de clase media (Subsidio para la Clase Media, sCM), y sus barrios se construían como proyectos separados. En tales circunstancias, se consideró que esa idea de vecindario homogéneo estaba entre los factores causantes de la segregación, por lo que era necesario modificarla. Esto derivó en el surgimiento de los pIs, barrios que incluían como mínimo $30 \%$ de hogares para ser adquiridas a través del Fsv y $30 \%$ a través del sCM, pudiendo el restante $40 \%$ ser vendido de forma directa a cualquier familia. La idea de estos vecindarios era disminuir los niveles de segregación socioeconómica por medio de la proximidad espacial de familias de diferente condición socioeconómica. Aunque el interés inicial de las empresas inmobiliarias por este tipo de barrio fue reticente, hoy el temor a la mezcla no parece ser obstáculo y la política de mixtura social parece estar consolidada (Sabatini \& Vergara, 2018). De hecho, los pIs concentran cerca del 36\% del presupuesto que anualmente el Ministerio de Vivienda y Urbanismo chileno (MINVU) destina a subsidios. ¿Cómo se rompió el temor a la integración que tenían las empresas inmobiliarias? Una posible respuesta proviene de los fondos que otorgan los subsidios vinculados a los PIs, los 
que han aumentado considerablemente desde 2007 hasta ahora, especialmente a partir de las modificaciones introducidas por el Ds 116, de 2014, y el Ds 19 de 2016 (Ruiz-Tagle \& Romano, 2019; Sabatini \& Vergara, 2018).

Sin embargo, y pese a las iniciativas que han tenido lugar en este ámbito, el incremento en el monto de los subsidios no parece estar motivando una mejor localización de los barrios de integración. Como registra el sitio web "tuvivienda", del MINVu, la mayoría de los PIs construidos al alero del Ds 116 se ubican en comunas periféricas y, en promedio, a 18,7 km en línea recta hasta centro de Santiago. La poca cantidad de proyectos erigidos en áreas pericentrales o centrales y la importante construcción en ciudades satélites contiguas al área metropolitana (Hidalgo, Santana \& Link, 2019; Sabatini \& Vergara, 2018), permiten sostener que los procesos de expulsión de vivienda social de las áreas centrales permanecen vigentes, aun con esta nueva política de vivienda.

El escaso énfasis locacional de los pis ha estado acompañado de una falta de visión urbanística respecto de los equipamientos con que deben contar estos lugares. Los primeros criterios de localización que regulaban los pis eran similares a los del Subsidio de Localización, el que -como fue documentado por Sabatini, Brain y Prieto (2011) - aplicaba a cualquier área de las ciudades, incluso fuera del área consolidada. Así, los PIs podían ser construidos a $1 \mathrm{~km}$ de un colegio, a $2,5 \mathrm{~km}$ de un centro de salud o a $1 \mathrm{~km}$ de un área verde, independientemente del tamańo de esta y sin importar la condición socioeconómica de las áreas que rodeaban dichas infraestructuras públicas. Aquellos criterios mínimos de localización han tenido pocas modificaciones en los Ds 116 y 19, más allá de algunos requerimientos de composición social del entorno; sin embargo, son requerimientos que solo poseen un carácter de sugerencia, lo que ha hecho que muchas empresas constructoras los omitan. Lo anterior ha derivado en que, tal como ocurría en 2007, hoy los PIs, en su gran mayoría, siguen siendo construidos en la periferia, con problemas de servicios y equipamientos, o bien localizados en áreas socialmente homogéneas, lo que no garantiza la resolución de otros tipos de segregación al interior de la ciudad (por ejemplo, la segregación educacional). Como se ha dicho previamente, estas medidas dan cuenta del poco enfoque urbanístico con que operan los pis, centrados solamente en medidas de proximidad socioeconómica.

Otra característica de la política de mixtura social en Chile ha sido la poca diversidad socioeconómica que promueve. Es sabido que los altos índices de concentración de riqueza y la importante movilidad social ascendente que ha mostrado el país en las últimas décadas al interior de familias de clase baja, han creado una pirámide de distribución especialmente ancha en los grupos medios bajos, que, por lo demás, no se diferencian tan radicalmente de la clase baja (Torche, 2005). Es allí donde se localiza la mezcla social que promueven los pIs: concentrada en clase media emergente y clase baja, junto a unas pocas familias de lo que podría llamarse clase media consolidada (Maturana, Vergara \& Romano, 2016; Sabatini \& Vergara, 2018). Es decir, se trata de una mixtura social restringida a grupos sociales que tienen capacidad de ahorro, pero que no son tan diferentes en términos de condición socioeconómica. La concentración de la mezcla social ha estado acompañada de una exclusión de este tipo de proyectos de aquellas familias de bajos ingresos sin 
capacidad de ahorro (Ruiz-Tagle \& Romano, 2019), como también de familias de altos ingresos que no se han visto atraídas por este tipo de iniciativas habitacionales.

Por tanto, aunque los PIs se proponen como una estrategia para solucionar los problemas de segregación y desigualdad urbana, entienden la mixtura social exclusivamente como proximidad socioeconómica; ponen allí su fundamento, sin establecer criterios adecuados de localización, equipamiento, tipo de mezcla y sin medidas concretas que fomenten la asociatividad entre residentes. Así visto, parece ser que la esperanza de sociabilidad pluriclasista que intentan promover los PIs está sostenida solo en la cercanía espacial, sin una estrategia urbana más amplia para fomentar el vínculo social al interior de los barrios o abordar desigualdades más estructurales, como la relacionada con el acceso a educación o salud, servicios clave en la calidad de vida de las personas. Cabe preguntarse, entonces: ¿no son las características sociales y territoriales del entorno del barrio y de la ciudad importantes para fomentar sociabilidades diversas desde el punto de vista socioeconómico? Lo que existe hasta ahora es una estrategia que intenta promover la proximidad y, desde ahí, impulsar procesos de sociabilidad pluriclasista; sin embargo, desde esta caracterización se desprenden las primeras dudas en relación a si este objetivo se consigue.

\section{El vínculo social, mixtura social y contexto urbano: la sociabilidad como fenómeno socioespacial y multiescalar}

En esta sección se complejiza la manera en que se produce la sociabilidad en un barrio de ingresos mixtos, entendido este fenómeno como "el proceso de interacción e intercambio, mediante el cual se materializa el encuentro con otros" (Link, Mora, Greene \& Figueroa, 2017, p. 11). En específico, se sostiene que la sociabilidad no es el simple resultado de la proximidad, sino que depende tanto de una variedad de condiciones sociales y espaciales que operan a nivel local (de barrio), como también de otras que lo hacen en escalas más lejanas (entorno y ciudad).

Un factor que influye en la sociabilidad de los vecindarios de ingresos diversos es la mixtura social que está alojada en ellos. De hecho, se debate respecto de si la diversidad es un obstáculo o no para la sociabilidad. Por un lado, se ha sostenido que en contextos de diversidad socioeconómica hay mayores posibilidades de encuentro, dado que allí se configura la necesidad de intercambios funcionales, como los laborales, que hacen que familias de diferente condición socioeconómica interactúen, se conozcan y reconozcan mutuamente como similares (Kleit \& Carnegie, 2011; Rasse, 2015; Sabatini \& Salcedo, 2007). Así, la mixtura social es una condición deseada, aspecto que se encuentra en la base de los llamados efectos de barrio, los cuales -junto a otras cosas- ayudan a inducir procesos de movilidad social, al menos en el plano económico, entre los más pobres (Sampson, Morenoff \& Gannon-Rowley, 2002). Por otro lado, se ha planteado también que en contextos de diversidad hay mayores posibilidades de surgimiento de nuevas formas de microsegregación cruzadas por el clasismo. En tal sentido, se ha postulado que la sociabilidad en barrios de ingresos mixtos se organizaría como las capas tectónicas en la tierra, donde las esferas de sociabilidad de los sujetos estarían estructuradas por su condición social (Butler \& Robson, 2003); esto es, mientras la clase baja tendería 
a presentar sociabilidades más ancladas cerca del barrio, las clases medias desplegarían un "habitus metropolitano" (Butler, 2002; Weber, 2007), con sociabilidades geográficamente más extendidas, desafiliando del vecindario -simbólica y funcionalmente- sus prácticas cotidianas (Watt, 2009). La investigación latinoamericana sobre sociabilidad en barrios ha confirmado las diferentes extensiones geográficas de las sociabilidades según clase (García, Carrasco \& Rojas, 2014; Link et al., 2017; Link \& Valenzuela, 2014; Marques, 2010), como también la importancia de la diversidad en el surgimiento de relaciones funcionales entre familias de diferente condición socioeconómica (Rasse, 2015; Sabatini \& Salcedo, 2007).

Pero la sociabilidad no solo depende de la mixtura social de un lugar, sino que también está influida por aspectos territoriales; en específico, por los espacios para el encuentro al interior del vecindario y también por la manera en que se hallan distribuidos diferentes equipamientos en el entorno y la ciudad. En este sentido, investigaciones han mostrado que una buena dotación del entorno minimizaría la tendencia metropolitana que tienen los vínculos de clase media, acercando sus prácticas cotidianas al lugar de residencia, creando así mayores posibilidades de encuentro con personas de la clase baja (Andreotti, Galès \& Fuentes, 2013; Dempsey, 2009). También la densidad y las funciones mixtas del espacio interior del barrio y de su entorno pueden producir mayor sociabilidad en este tipo de vecindarios, lo que explica la importancia de los espacios públicos para fomentar el vínculo social (Dempsey, 2009; Raman, 2010; Talen, 2002). Ahora bien, los espacios de encuentro entre clases serían negociables; es decir, el contacto pluriclasista no sería tolerado en todas las esferas de la vida cotidiana por parte de la clase media (Bacqué, Charmes \& Vermeersch, 2014). Se producirían así "salidas parciales" del lugar por parte de la clase media (Andreotti, Galès \& Fuentes, 2013), que controlarían su tendencia hacia la desafiliación.

Junto a la dotación de espacios públicos, otro aspecto relevante en la sociabilidad en contextos socioeconómicamente diversos es la manera en que se configura el espacio social en el entorno y la ciudad. En ciudades marcadas por la segregación socioeconómica y la desigual distribución de equipamiento urbano, como Santiago y otras grandes ciudades latinoamericanas, los proyectos de mixtura social tendrían menos capacidad para inducir sociabilidades diversas a escala de barrio, especialmente cuando estos se construyen en entornos populares. Cuando el entorno es socioeconómicamente homogéneo, la disposición de familias de altos ingresos a habitar barrios mezclados disminuye y lo mismo ocurre cuando ese entorno no está bien dotado (Sabatini, Rasse, Mora \& Brain, 2012). Esto último -la pobreza de dotación- lleva también a que las familias deban desplazarse largos trayectos al trabajo o lugares de estudio, lo que naturalmente disminuye el tiempo que se pasa en la residencia y, con ello, las posibilidades de encuentro (Maturana \& Horne, 2016). Así, la manera en que se organiza el espacio social de la ciudad-que, en el caso de Santiago, concentra buena parte de su clase alta en el llamado "cono de alta renta”, es decir, lejano a los lugares donde suelen construirse los PIS- podría operar como un obstáculo para el encuentro pluriclasista en proximidad.

En definitiva, los diferentes factores revisados permiten sostener que la sociabilidad al interior de un barrio de ingresos mixtos no solo se juega por la proximidad 
de clases que hay en él, lo que es en el fondo un aspecto social. La mirada debe expandirse también a factores espaciales. Adicionalmente, la sociabilidad debe ser entendida como multiescalar, en el sentido de que este fenómeno no depende únicamente del barrio, sino también, y estrechamente, de las características socioterritoriales de otras escalas al interior de la ciudad: del entorno y, por cierto, de otros distritos de la ciudad y de cómo en ellos se distribuyen los equipamientos y los diferentes grupos socioeconómicos.

\section{Estrategia metodológica y casos de estudio}

Para entender cómo las condiciones de mixtura y de entorno sobre las cuales los PIS son construidos influyen en la sociabilidad de los sujetos que los habitan, se desplegó en este estudio una metodología mixta. En su dimensión cuantitativa se analizó el nivel de sociabilidad anclada en el barrio y otras escalas urbanas, como también el nivel de diversidad socioeconómica que adquirían los vínculos de los residentes de los PIs. Para ello se empleó el llamado análisis de redes sociales egocéntricas, "definidas desde el punto de referencia de un agente social determinado (Ego), directamente conectado a otros agentes sociales" (Rincón, 2015, p. 22), junto a un generador de posiciones, lo que permitió vincular a Ego y sus contactos con una determinada posición social (Espinoza \& Durston, 2013). Esa vinculación se hizo de forma indirecta a través del tipo de trabajo/profesión que se tiene, un indicador muy usado en países desiguales y con consumo masificado como Chile (Espinoza \& Barozet, 2009; Link, Valenzuela \& Fuentes, 2015). Para relacionar posición social y posición laboral se adjudicó a la clase alta las labores directivas de administración pública y empresas, y algunas profesiones científicas; se identificó a la clase media consolidada con profesionales y algunos técnicos de nivel medio; la clase media emergente fue vinculada a técnicos de nivel medio, empleados de oficina, trabajadores de servicios, vendedores, trabajadores calificados y algunas actividades de agricultura; y la clase baja, a labores de operación de maquinarias, algunos servicios, agricultura y empleos no calificados (Link et al., 2015). De esta forma, la pregunta inicial que se hizo a cada encuestado fue: ¿Conoce a alguna persona que tenga x profesión?, recogiéndose datos sobre los contactos en 76 actividades laborales diferentes. Se aplicaron 66 encuestas en dos PIs representativos, recolectándose información de 1658 contactos. Se solicitó también información sobre la residencia de los contactos, en el entendido de que las relaciones sociales se extienden por diferentes escalas (Kleit \& Carnegie, 2011): barrio, entorno y otras comunas de Santiago. La combinación de estas variables (ocupación y lugar de residencia) permitió descifrar si el barrio estaba produciendo o no sociabilidades más heterogéneas (Tabla 1). 
TABLA I Caracterización socioeconómica de la muestra de encuestas y entrevista

\begin{tabular}{|c|c|c|c|c|c|}
\hline CARACTERIZAC & ÓN SOCIOECONÓMICA & & & $\begin{array}{r}\text { EI } \\
\text { SEMIE }\end{array}$ & $\begin{array}{l}\text { TA } \\
\text { URADA }\end{array}$ \\
\hline & & SA & JV & JV & SA \\
\hline $\mathrm{C}_{0} \mathrm{O}$ & Hombre & 11 & 14 & 6 & 6 \\
\hline & Mujer & 22 & 19 & 14 & 15 \\
\hline & $18-26$ & 1 & 0 & 0 & 0 \\
\hline & $26-40$ & 19 & 18 & 11 & 12 \\
\hline Edad & $40-50$ & 5 & 8 & 5 & 6 \\
\hline & $50-65$ & 6 & 5 & 3 & 2 \\
\hline & 65 & 2 & 2 & 1 & 1 \\
\hline & Básica incompleta & 3 & 0 & 2 & 0 \\
\hline & Básica completa & 2 & 1 & 1 & 0 \\
\hline & Media incompleta & 7 & 5 & 3 & 1 \\
\hline Escolaridad & Media completa & 15 & 10 & 10 & 9 \\
\hline & Técnica superior & 5 & 10 & 3 & 7 \\
\hline & Universitaria & 3 & 7 & 1 & 4 \\
\hline & Posgrado & 0 & 0 & 0 & 0 \\
\hline & Clase baja & 21 & 11 & 13 & 7 \\
\hline Clocescis & Clase media emergente & 7 & 14 & 5 & 9 \\
\hline & Clase media consolidada & 5 & 8 & 2 & 5 \\
\hline & Clase alta & 0 & 0 & 0 & 0 \\
\hline & Fondo solidario & 14 & 14 & 9 & 8 \\
\hline & Subsidio clase media & 13 & 13 & 7 & 9 \\
\hline Tipo de propiedad & Compra directa & 1 & 1 & 1 & 1 \\
\hline & Arrendamiento & 2 & 3 & 2 & 1 \\
\hline & Allegado & 3 & 2 & 1 & 2 \\
\hline
\end{tabular}

JV: PROYECTO DE INTEGRACIÓN SOCIAL JUVENCIO VALLE. SA: PROYECTO DE INTEGRACIÓN SOCIAL SAN ALBERTO DE CASAS VIEJAS

FUENTE: ELABORACIÓN PROPIA

La dimensión cualitativa del trabajo buscó entender cómo la dotación del barrio y del entorno en que los pis son construidos influyen en la sociabilidad de los residentes. Específicamente se analizaron las prácticas sociales y el uso que los sujetos daban a diferentes puntos de encuentro con los que contaban los vecindarios y su entorno: plazas, parques, almacenes, ferias, escuelas, hospitales y sedes comunitarias. Para ello se desarrolló una entrevista semiestructurada que fue aplicada a 33 residentes. Usando una técnica de bola de nieve, la muestra estuvo definida por 
saturación teórica de los datos, que fueron analizados a partir de una codificación abierta en el software Atlas.ti.

Para la investigación fueron empleados dos pis de la periferia sur de Santiago: San Alberto de Casas Viejas (sA), localizado en el sector de Casas Viejas en el piemontino de Puente Alto; y Juvencio Valle (Jv), ubicado en el límite de las comunes de San Bernardo, El Bosque y La Cisterna, ambos los primeros pis construidos en la ciudad. La selección de dichos casos estuvo basada, por una parte, en un criterio de antigüedad, que permitiera cierta estabilidad en la forma en que se desarrolla la sociabilidad en ellos; y por otra, en un criterio de escala urbana metropolitana que favoreciera la comparación, excluyendo a otros pIs construidos en ciudades intermedias o pequeńas y cuya relación con la ciudad en la que se localizan es diferente. Finalmente, la composición social de ambos barrios es representativa de los tipos de mezcla que proponen los pIs a nivel nacional; esto es, concentrada en grupos de clase baja y media baja (Maturana \& Horne, 2016; Sabatini \& Vergara, 2018). A pesar de estos puntos en común, los PIs en estudio se ubican en entornos diferentes. Mientras SA lo hace en un área de expansión urbana, Jv se encuentra en un sector consolidado de la ciudad, lo que implica que los barrios están insertos en entornos desigualmente dotados. En efecto, en sA solo es posible encontrar tres colegios y un centro de salud familiar en el área circundante, en tanto que en Jv hay al menos dos supermercados, varios colegios, un hospital y una de las más grandes ferias populares que funciona diariamente en el sur de Santiago. Ambos barrios y sus entornos presentan, entonces, diferencias en términos de dotación de espacios públicos, lo que permite evaluar también el peso que tiene la dotación de servicios sobre la sociabilidad del lugar (Figura 1).

\section{FIgURA I | Dotación de los casos de estudio}

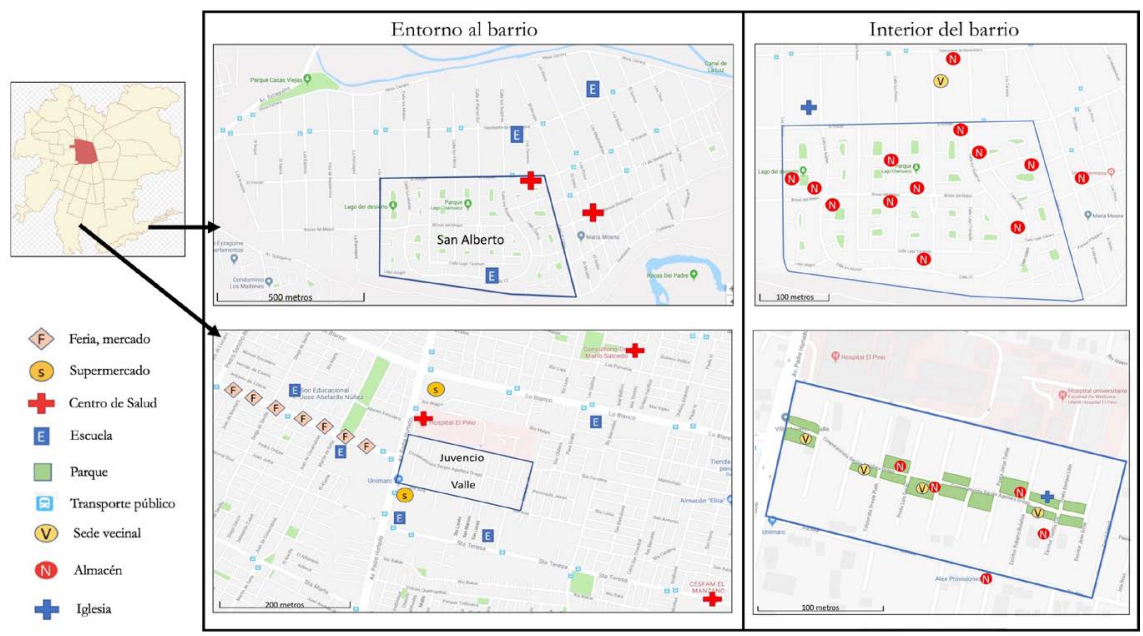

FUENTE: ELABORACIÓN PROPIA SOBRE IMÁGENES DE GOOGLE MAPS 


\section{La sociabilidad intrabarrio: escaso contacto y homogeneidad socioeconómica}

Las políticas de mixtura trabajan sobre la premisa de que en contextos de proximidad entre clases sociales habrá mayor contacto entre ellas, generando así mayor diversidad en los vínculos sociales. Sin embargo, según han advertido diferentes trabajos locales (Maturana \& Horne, 2016; Ruiz-Tagle, 2016; Sabatini \& Brain, 2008; Vergara, 2019), la proximidad no produce necesariamente mayores niveles de interacción, situación que es corroborada por esta investigación. Los resultados muestran que, en promedio, el 23,1\% del total de los contactos de los residentes se localiza dentro del barrio. Dicho valor es bastante menor al encontrado por Link et al. (2017) en barrios de vivienda social chilenos, donde alrededor del 50\% del volumen de sociabilidad se localiza en el vecindario, pero se acerca más a lo que García et al. (2014), Marques (2010) y Kleit y Carnegie (2011) detectaron en barrios de clase media en Chile, Brasil y Estados Unidos, respectivamente.

Con independencia de su condición socioeconómica, los residentes entrevistados declaran que el contacto entre vecinos es esporádico y suele remitirse exclusivamente al saludo cordial y conversaciones poco profundas. Son pocos los habitantes que interactúan con otros vecinos de manera cercana. Esto último ocurre especialmente con quienes ocupan cargos dentro de la dirigencia vecinal, aunque también ellos observan escaso interés por parte de los residentes en cuanto a participar en las actividades o reuniones que se realizan para tratar temas comunes. Así, el ambiente social dentro de los pis estudiados se caracteriza por el poco interés en el contacto, tal como encontraron previamente Maturana y Horne (2016) y Maturana, Vergara y Romano (2016). Aquella falta de contacto es una situación que acomoda a gran parte de los entrevistados, porque les permite mantener y resguardar su vida privada. Sin embargo, quienes desean mayor interacción criticaron la falta de actividades comunes impulsadas por el MINvU, la Municipalidad u otra institución para que los vecinos se conocieran. Esto fue considerado como un elemento crucial para la interacción social al interior del vecindario y así también lo ha comenzado a considerar el MINVU desde 2016, cuando estableció un "bono de participación" de alrededor de 141.000 CLP (175 uSD), que se suma al subsidio otorgado a cada persona que participe en planes de integración social planificados por las entidades desarrolladoras de los pis.

A pesar de lo anterior, los resultados revelan que la sociabilidad que tiene lugar al interior del barrio tiende a ser poco diversa desde el punto de vista socioeconómico, especialmente en JV. Los datos contenidos en la Tabla 2 confirman la casi total ausencia de clases altas en ambos vecindarios. El único contacto de clase alta detectado fue registrado en SA, y se trató de un integrante del Concejo Municipal local, por lo que su posición social podría ser incluso temporal. La falta de clases altas en los pis puede ser uno de los principales obstáculos para los objetivos que persiguen estos barrios y da soporte a la idea de que estos lugares solo promueven una mixtura social confinada en clases medias bajas y bajas (Maturana, Vergara \& Romano, 2016; Sabatini \& Vergara, 2018). De hecho, en promedio, el 85,6\% de los vínculos localizadas en ambos vecindarios se despliega con personas de estos grupos, por lo que en ellos también hay poca presencia de clase media consolidada. 
TABLA 2 Distribución socioeconómica de los contactos al interior del barrio (en porcentajes)

\begin{tabular}{|l|c|c|c|}
\hline $\begin{array}{c}\text { CLASE SOCIAL DE LOS } \\
\text { CONTACTOS EN EL BARRIO }\end{array}$ & AMBOS BARRIOS & SA & JV \\
\hline Clase alta & 0,5 & 1,0 & 0 \\
\hline Clase media consolidada & 13,9 & 20,3 & 6,3 \\
\hline Clase media emergente & 39,8 & 40,6 & 38,8 \\
\hline Clase baja & 45,8 & 38,1 & 54,9 \\
\hline
\end{tabular}

FUENTE: ELABORACIÓN PROPIA

Los resultados de la escala barrio no parecen tan alentadores en términos de promover sociabilidades más diversas, porque, aunque los pIs quiebren la antigua homogeneidad socioespacial derivada de la construcción de barrios con un solo tipo de subsidio, parece aún insuficiente en términos de promover relaciones sociales diversas desde el punto de vista socioeconómico. La lógica de integración de subsidios para clase baja y media baja, sumada a la venta directa para clase media consolidada y alta con la que trabajan estos barrios, no parece rendir buenos frutos en términos de fomentar sociabilidades más diversas a nivel de vecindario. Un aspecto determinante en las tendencias señaladas es la composición social del lugar. De hecho, como se observa en la Tabla 2, la distribución socioeconómica de la sociabilidad sigue un patrón similar respecto a la composición social de estos lugares, incorporando levemente más contacto con clase media consolidada en SA, allí donde Sabatini et al. (2014), en una investigación previa, encontraron mayor presencia de este grupo.

La diversidad socioeconómica del lugar es un aspecto crucial en la operatoria de los efectos de barrio, que son los que persiguen las políticas de mixtura social. Sin embargo, en un contexto marcado por la escasa diversidad socioeconómica, los beneficios funcionales de aquello tienden a ser menores (Kleit \& Carnegie, 2011; Sampson et al., 2002). De hecho, la mayor parte de los vínculos existentes al interior del barrio fueron clasificados como "conocidos", desde los cuales, a pesar de que se mantenga un vínculo débil, es difícil obtener acceso a recursos sociales y oportunidades. Asimismo, no fue posible encontrar opciones de trabajo estable al interior de los barrios. Según se registró, solo esporádicamente aparecen algunos trabajos manuales entre residentes. La transversalidad socioeconómica de estos trabajos sugiere que la escasez de oferta dentro del vecindario no se produce por la estigmatización de un grupo específico. Esto puede tener implicancias importantes, porque en un contexto de diversidad donde se incluya más clase media consolidada y alta se podrían originar mayores posibilidades de trabajo en el vecindario para familias de escasos recursos, como lo han encontrado otros estudios en Chile (Rasse, 2015; Sabatini \& Salcedo, 2007). Sin embargo, lo que juega en contra en la experiencia de los pis es la falta de diversidad social del barrio. Es aquello lo que explica la poca capacidad de estos vecindarios para crear trabajos. 


\section{La sociabilidad más allá del barrio: entre el localismo y el "habitus metropolitano"}

Los resultados sugieren que la mayoría de los contactos que poseen los habitantes de los PIs se localizan en el entorno del barrio (38\%); esto es, en la misma comuna de Puente Alto para los residentes de sa y en las comunas de San Bernardo, El Bosque y La Cisterna para los habitantes de Jv. Un porcentaje importante de los contactos también habita en otras comunas de la Región Metropolitana (33\% de los contactos). Ambas escalas concentran un mayor número de contactos que las alojadas en el barrio. Pero la tendencia de distribución espacial de la sociabilidad cambia levemente cuando se analiza por caso de estudio. Mientras el entorno es la escala que mayor volumen de sociabilidad concentra para los residentes de JV, otras comunas de Santiago lo son para los habitantes de SA. Se registra además que, en el primero de estos casos, la escala barrio y el entorno concentran más del 68\% de los contactos totales, mientras que en sa la cifra alcanza el 55\%. Estos antecedentes permiten argumentar que los vínculos sociales de los residentes de Jv tienden a ser más locales, mientras que los de sa están más extendidos espacialmente, lo que sugiere que en este último barrio se desarrollan prácticas más metropolitanas. Los contactos registrados en regiones diferentes a la metropolitana no tienen trascendencia estadística, como tampoco la tienen las relaciones con personas en el extranjero. Esto podría relacionarse con que los residentes de los PIs han vivido mayoritariamente en Santiago, y también con la falta de población migrante en los barrios.

TABLA 3 | Distribución socioeconómica de los contactos en el entorno y otros lugares de Santiago (en porcentajes)

\begin{tabular}{|l|c|c|c|c|c|c|}
\hline \multirow{2}{*}{$\begin{array}{c}\text { CLASE SOCIAL DE } \\
\text { LOS CONTACTOS }\end{array}$} & \multicolumn{2}{|c|}{ AMBOS BARRIOS } & \multicolumn{2}{c|}{ SA } & \multicolumn{2}{c|}{ JV } \\
\cline { 2 - 7 } & EL BORNO & $\begin{array}{c}\text { EN OTRO } \\
\text { LUGAR DE } \\
\text { SANTIAGO }\end{array}$ & $\begin{array}{c}\text { EN TORNO } \\
\text { AL BARRIO }\end{array}$ & $\begin{array}{c}\text { EN OTRO } \\
\text { LUGAR DE } \\
\text { SANTIAGO }\end{array}$ & $\begin{array}{c}\text { EN TORNO } \\
\text { AL BARRIO }\end{array}$ & $\begin{array}{c}\text { EN OTRO } \\
\text { LUGAR DE } \\
\text { SANTIAGO }\end{array}$ \\
\hline Clase alta & 4,5 & 15,7 & 4,9 & 17,7 & 3,8 & 11,0 \\
\hline Clase media consolidada & 25,4 & 34,7 & 31,6 & 34,6 & 17,0 & 35,0 \\
\hline Clase media emergente & 33,2 & 30,0 & 31,3 & 28,9 & 35,8 & 32,5 \\
\hline Clase baja & 36,9 & 19,6 & 32,2 & 18,8 & 43,4 & 21,5 \\
\hline
\end{tabular}

FUENTE: ELABORACIÓN PROPIA

La Tabla 3 muestra la distribución socioeconómica de los contactos de residentes de los pis en escalas diferentes al barrio. Comparando estos resultados con los de la Tabla 2, se puede argumentar que, a medida que los contactos se alejan del barrio, la diversidad socioeconómica de los mismos tiende a aumentar. El patrón de dis-tribución socioeconómica de los vínculos comienza a cambiar en el entorno. Así, los contactos de clase baja que poseen los habitantes de JV y sA disminuyen su pro-porción en comparación al barrio, y esa tendencia continúa hasta la escala metro-politana. Ocurre lo mismo con los contactos de clase media emergente en ambos 
casos de estudio. Simultáneamente, los contactos con clase media consolidada se incrementan en la escala entorno, mientras que los de clase alta irrumpen notablemente en la escala metropolitana en los dos vecindarios. Por consiguiente, es fuera del barrio y del entorno donde es más probable encontrar contactos de clase alta y media consolidada, es decir, contactos más diversos socioeconómicamente.

Una posible explicación a esta tendencia es que, a medida que nos alejamos del barrio, encontramos, casi naturalmente, mayor disponibilidad de clases sociales debido a que la población incrementa y, por tanto, hay más probabilidad de contacto pluriclasista en esa escala territorial que en el barrio. Sin embargo, aunque esto sea válido, hay que hacer notar que la diversidad socioeconómica inducida en la escala metropolitana se encuentra segmentada espacialmente. Tal como muestra la Figura 2, las clases alta y media consolidada suelen encontrarse en comunas alejadas de los pis en estudio, fundamentalmente de Jv (Vitacura, Las Condes, La Reina, Providencia, Santiago, Pirque, La Florida), a la vez que se concentran sea en el llamado "cono de alta renta de la ciudad" o bien en comunas periféricas con atributos naturales y paisajísticos. Por el lado de los contactos de clase media emergente y baja, estos aparecen más dispersos en toda el área metropolitana, pero la tendencia en ambos vecindarios parece clara: se encuentran localizados en comunas periféricas y que tradicionalmente han albergado vivienda social. Estos resultados se relacionan con el patrón de distribución que las diferentes clases sociales tienen al interior de la ciudad y que configuran un mapa de sociabilidad segregado, el cual tiende a limitar el desarrollo de sociabilidades diversas en escalas más locales, pese a que en Santiago el patrón de segregación esté transformándose (Sabatini, Cáceres \& Cerda, 2001).

FIGURA 2 | Distribución comunal de los contactos según su clase social
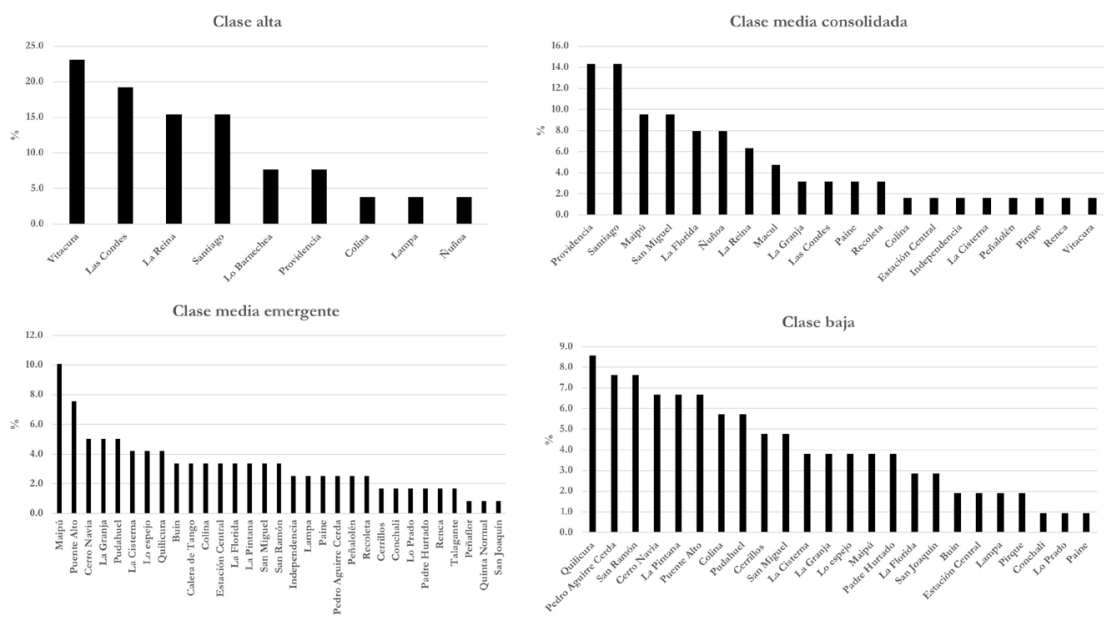

FUENTE: ELABORACIÓN PROPIA 
Este último resultado viene a cuestionar la localización de los PIs. ¿Cómo conseguir sociabilidades más diversas si el barrio no lo es y si la ciudad presenta importantes niveles de segregación? Aquí es donde podría adquirir importancia el entorno del barrio. No obstante, en este aspecto los PIs se enfrentan a otro problema. Como fue descrito, estos barrios tienden a estar localizados en zonas periféricas y cercanas a barrios segregados. SA es un caso atípico, ya que se localiza en un área de desarrollo inmobiliario de alto estándar y eso podría estar actuando para promover una sociabilidad de entorno con mayor presencia de clase media consolidada, como indica la Tabla 3. De hecho, se corroboró que los propios residentes del barrio perciben el entorno como un lugar de ingresos mixtos, cuestión que, a su entender, es clave en el desarrollo de sociabilidades más diversas. Esa mixtura no forma parte de la percepción de entorno que tienen los residentes de JV; por el contrario, hay acuerdo entre los entrevistados de ese lugar respecto a que los barrios adyacentes son homogéneos, de clase baja y media baja.

Los resultados muestran también la existencia de diferencias respecto a la distribución socioeconómica que la sociabilidad de los encuestados tiene fuera del barrio. Mientras la sociabilidad de los encuestados asociados a clase baja tiende a ser más homogénea y estar más anclada en el entorno, la sociabilidad de la clase media emergente y consolidada es más diversa y más extendida espacialmente. Esto confirma hallazgos de otros estudios en relación a que las sociabilidades de los grupos pobres tienden a ser más locales, mientras que las clases medias poseen una sociabilidad más expandida geográficamente (García et al., 2014; Link \& Valenzuela, 2014; Link et al., 2017), lo que sugiere que esta última tiende a desarrollar prácticas más metropolitanas dentro de la ciudad (Butler, 2002; Webber, 2007).

Estos resultados muestran que el grado de diversidad socioeconómica que posee la sociabilidad de una persona está estructurada socioespacialmente; es decir, primero por su propia condición socioeconómica, y segundo, por la configuración del espacio social que tiene la ciudad en que habita. Este último punto es clave, por cuanto una estructura de ciudad segregada como Santiago parece hacer más difícil la consecución de sociabilidades pluriclasistas, especialmente cuando los PIs se construyen mayoritariamente en una periferia popular.

\section{La importancia de los equipamientos del lugar: ¿cómo minimizar el habitus metropolitano?}

Los resultados de la anterior sección sugieren que existen diferencias en términos de la extensión geográfica de los vínculos, dependiendo de la clase social de las personas que habitan los PIs. Sin embargo, en esta sección se muestra cómo servicios y lugares existentes en el entorno del barrio tienen capacidad para producir encuentros entre vecinos de diferentes clases sociales, promoviendo así "salidas parciales" del lugar. En este sentido, ciertas intervenciones espaciales tienen capacidad para modificar algunas tendencias de la sociabilidad.

La literatura internacional ha sugerido que los equipamientos y espacios de encuentro en el entorno del barrio son importantes para asegurar la integración con otros vecindarios y con sus residentes, evitando así los procesos de fragmentación 
socioespacial de la ciudad. Un espacio clave para ello son los colegios, que pueden ayudar a atar al lugar los habitus metropolitanos que despliegan algunas clases medias (Bacqué et al., 2014; Butler \& Robson, 2003; Méndez \& Gayo, 2018). Sin embargo, las entrevistas mostraron que las escuelas no se constituyen como un lugar de encuentro entre residentes de diferentes niveles socioeconómicos. Esto fue especialmente claro en JV. Allí los residentes de menores ingresos suelen enviar a sus hijos a las escuelas públicas o subvencionadas del sector, porque la educación en ellas no tiene costo y porque son cercanas. Aunque dichas escuelas no son prestigiosas, la decisión de enviarlos allí es esencialmente práctica. Diferente es la posición de residentes de clase media consolidada, que suelen enviar a sus hijos a establecimientos localizados fuera del entorno. Entre ellos existe la idea de que aquellos colegios localizados en el vecindario tienen malos rendimientos académicos y problemas de disciplina, sensación que se refuerza por la estigmatización social del área. Por esa razón, prefieren evitar el barrio en términos educacionales, disminuyendo así la capacidad de este espacio para producir sociabilidades pluriclasistas. Como señala una de las personas entrevistadas:

A él [hijo] lo tengo en un colegio aquí cerca, este año ya le estoy buscando colegios para Providencia. Mi mamá me dice que estoy loca, que es un viaje increíble, pero que lo vea, vea si se siente cómodo (...) si a él le gusta, me lo llevo al colegio en Providencia. (Entrevistada, clase media consolidada, Jv)

Las palabras anteriores confirman la presencia de habitus metropolitanos en los PIS, particularmente asociados a la clase media consolidada, como también la centralidad de los colegios en los procesos de segregación socioeconómica de Santiago (Méndez \& Gayo, 2018; Stillerman, 2016). Ambas tendencias fueron reconocidas también en el uso de otros espacios públicos, como los centros de salud que rodean los barrios. Al igual que ocurre en los colegios, lo público suele ser más usado por residentes de clase baja y media baja, mientras la clase media consolidada se inclina por el uso de servicios privados. Así, este último grupo privilegia la exclusividad de los lugares por sobre la cercanía de los servicios, lo que resulta fundamental en la formación de su identidad de clase (Castillo, 2016; Méndez, 2008). El abandono de lo público por parte de un sector de la clase media hace que los servicios del Estado pierdan capacidad para promover sociabilidades mixtas, a pesar de que el entorno a los barrios cuente con este tipo de infraestructura.

A pesar de lo anterior, un resultado llamativo fue que en ambos barrios se encontraron algunos espacios de encuentro común que trascienden las diferencias socioeconómicas de los residentes: la feria y las plazas. En relación a la feria, una buena porción de los residentes concurre al menos una vez por semana a ese lugar, reuniéndose allí con familiares, amigos y conocidos que viven tanto fuera como dentro del barrio. Asimismo, establecen amistades o relaciones sociales con los vendedores del lugar, quienes en su mayoría también residen en el entorno y, en algunos casos, en los mismos barrios. En el caso de las plazas, su uso es intenso en JV, encontrándose buena parte de las tardes ocupadas por actividades de la Junta de Vecinos o simplemente usadas para que los nińos jueguen o las mascotas paseen. En aquellas actividades se producen encuentros entre vecinos de los pIs, aunque, 
a diferencia de la feria, en las plazas el encuentro tiende a ser más homogéneo desde el punto de vista socioeconómico, dependiendo esto de la composición de los vecindarios. Así, las ferias y las plazas constituyen espacios públicos con capacidad de articular y promover sociabilidades en el entorno y el barrio.

Lo anterior lleva a sugerir que un barrio y entorno bien dotados de servicios podrían amortiguar en alguna medida las tendencias metropolitanas propias de la clase media consolidada, produciendo así espacios de encuentro entre clases sociales y, con ellos, dando lugar a salidas parciales y no totales del lugar (Andreotti et al., 2013; Bacqué et al., 2014). Sin embargo, en el caso chileno la sociabilidad inducida por espacios públicos como colegios o centros de salud estaría más bien segmentada socioeconómicamente, siendo solo la feria y las plazas los espacios públicos con capacidad para producir sociabilidades potencialmente más diversas. Las razones de dicha diferencia se vinculan, primero, a la estigmatización y marginación que se ha hecho de lo público y que ha llevado a la clase media -que busca construir una identidad social alejada de todo lo estigmatizado (Castillo, 2016; Méndez, 2008)al abandono general de los servicios estatales. Y segundo, a la superficialidad con la que se produce el contacto en cada lugar, siendo en tal sentido más sensible la escuela, por cuanto allí se puede intervenir y formar conductas que en un contexto de estigmatización son evitadas, no así en la feria o en la plaza, donde el contacto es más temporal y funcional. A pesar de que estos espacios pueden amortiguar tendencias hacia la desafiliación funcional de la clase media consolidada, los vínculos que surgen desde ella no son necesariamente diversos desde el punto de vista socioeconómico. Dadas las características de la composición socioeconómica de los PIs y de sus entornos, la sociabilidad que emerge desde ferias y plazas se limita mayoritariamente a personas de clase media emergente y clase baja, tal como se mostró en la sección anterior. Por lo tanto, aunque el barrio y el entorno tengan buena dotación y equipamientos, si no existe diversidad en el barrio y en el entorno, pareciera ser difícil que los PIs impulsen vínculos socioeconómicamente más diversos. Nuevamente, la estructura segregada del espacio social de Santiago impone dificultades para producir sociabilidades socioeconómicamente diversas.

\section{Conclusiones}

Este trabajo buscó entender cómo las condiciones de mixtura y de entorno sobre las cuales los pIs son construidos influyen en la sociabilidad de sujetos que los habitan. Los resultados sugieren que la diversidad social que proponen estos vecindarios es insuficiente para promover sociabilidades que incorporen, por ejemplo, grupos de clase alta, a la vez que incluyen pocos integrantes de la clase media consolidada. Así, la sociabilidad parece estar restringida a familias de clase media emergente y baja, lo que podría limitar en el mediano y largo plazo el desarrollo de efectos de barrio positivos. De hecho, el argumento de que la mixtura social podría generar nuevas oportunidades laborales en las cercanías del barrio tiene poco respaldo en los casos analizados. Con respecto a las condiciones de entorno en las cuales se están construyendo estos vecindarios, parece haber deficiencias. Dado que la mayoría de los PIs se erigen en entornos homogéneos, mayormente compuestos de clase media 
emergente y baja, no pueden emanar sociabilidades mixtas desde lo que rodea al barrio. La estructura de distribución socioeconómica de Santiago cumple también un papel condicionante en la diversidad social de la sociabilidad, lo que establece un desafío mayor en términos de la localización de los pis. Finalmente, algunos espacios de encuentro ubicados alrededor de los barrios -como las plazas y las ferias- parecen relevantes para amortiguar las tendencias de habitus metropolitano observados en residentes de clase media consolidada.

Estos hallazgos complejizan la mirada en torno a la sociabilidad en vecindarios de ingresos mixtos, mostrando que este es un fenómeno que va más allá de la proximidad social. Los resultados permiten afirmar que, primero, la sociabilidad del vecindario es un fenómeno socioespacial. Es decir, el vínculo social depende de una serie de dinámicas sociales -como, por ejemplo, el grado de diversidad de los lugares-, pero también de aspectos territoriales, como la disponibilidad de espacios de encuentro en los entornos. Y, segundo, que la sociabilidad debe ser entendida como un fenómeno multiescalar. El vínculo social en un barrio de ingresos mixtos no se establece solo por aspectos sociales y físicos propios del vecindario, sino también por aquello que está fuera de este, en el entorno y, por cierto, también por la manera en que se organiza el espacio social al interior de la ciudad. Esto último es medular en el desarrollo de una política de barrio, la que no puede ser planeada solo como una intervención específica, sino también en un sentido de escala urbana más amplia.

La adopción de un enfoque socioespacial y multiescalar para entender la sociabilidad de barrios de ingresos mixtos permite sugerir al menos tres líneas de acción para mejorar el desarrollo de los PIs, aspectos que también pueden ser replicables para otras políticas pro mezcla social que comienzan a ser desarrolladas en ciudades latinoamericanas.

- Primero, aumentar el grado de diversidad que proponen los PIs. Si se quiere trabajar en función de la operación de efectos de barrio, es necesario que existan posibilidades de encuentro más diversas socioeconómicamente dentro del vecindario. Ello podría aumentar las posibilidades de acceder a redes portadoras de otros recursos sociales, las que hoy no parecen estar disponibles en los PIs.

- Segundo, asegurarse de que el entorno de los pis sea un lugar diverso desde el punto de vista socioeconómico, dejando de replicar un patrón de localización periférico y homogéneo. Si bien existe hoy una norma que regula la construcción de PIs en lugares con menos de $50 \%$ de vivienda social en el entorno, es una sugerencia y no una obligación, por lo que muchas empresas inmobiliarias la omiten. Conseguir localizaciones en áreas diversas requiere mucho dinero y, por tanto, lleva a sugerir un cambio mayor en la manera en que se financia la política habitacional chilena, donde parece urgente incorporar, por ejemplo, métodos para capturas de plusvalías, modificaciones en los sistemas de impuestos territoriales o bien la utilización de bancos de terrenos públicos (Ruiz-Tagle, Labbé, Rocco, Schuster \& Muñoz, 2019).

- Finalmente, se requieren diseños de barrio y localizaciones con espacios que promuevan el encuentro entre personas de diferente condición económica. El 
barrio y su entorno no solo deben ser lugares residenciales, sino también promover usos mixtos. Los resultados de este trabajo muestran cuán importantes son, por ejemplo, las plazas y las ferias. Esto implica un cambio de enfoque en la política, la que debe pasar de estar soportada exclusivamente en el apoyo individual a través de subsidios, a una que apoye también activamente los lugares, con infraestructura que favorezca el encuentro (Sabatini \& Vergara, 2018). El apoyo a los lugares, materializado en la construcción de espacios de encuentro pluriclasista podría ayudar a crear una base valorativa común que facilite reconocer al otro como un semejante. Una inversión más fuerte en lo público podría también derribar ciertos prejuicios de las clases medias hacia servicios escolares o de salud, revalorizándolos como espacios de encuentro en diversidad. Esto parece clave para crear cohesión social en una sociedad que atraviesa una crisis como la que vive actualmente Chile.

Este conjunto de sugerencias apela a que la política de mixtura social reconozca como base que la mezcla en sí misma no es suficiente. Se necesitan a la vez intervenciones espaciales, aspecto crucial en momentos en que la Ley de Integración se discute en el Congreso; y también una mirada territorial más amplia y relacional respecto a la manera en que se configura la sociabilidad en barrios de mixtura social. De lo contrario, la actual retórica a favor de la mixtura -como también han argumentado Ruiz-Tagle y Romano (2019) - no sería un real intento para resolver los problemas de segregación de la ciudad, sino más bien una estrategia para darle continuidad, con subsidios públicos, al negocio inmobiliario.

\section{Referencias bibliográficas}

Andreotti, A., Le Galès, P. \& Fuentes, F. (2013). Controlling the urban fabric: the complex game of distance and proximity in European upper-middle-class residential strategies. International Journal of Urban and Regional Research, 37(2), 576-597. https://doi. org/10.1111/j.1468-2427.2012.01177.x

Bacqué, M., Charmes, E. \& Vermeersch, S. (2014). The middle class 'at home among the poor'. How social mix is lived in Parisian suburbs: between local attachment and metropolitan practices. International Journal of Urban and Regional Research, 38(4), 1211-1233. https://doi.org/10.1111/1468-2427.12130

Butler, T. (2002). Thinking global but acting local: the middle classes in the city. Sociological Research Online, 7(3), 1-19. https://doi.org/10.5153\%2Fsro.740

Butler, T. \& Robson, G. (2003). London calling: the middle classes and the remaking of inner London. Oxford: Berg Publishers.

Castillo, M. (2016). Fronteras simbólicas y clases medias. Movilidad social en Chile. Perfiles Latinoamericanos, 24(48), 213-241. http://dx.doi.org/10.18504/p12448-009-2016

Comisión Económica para América Latina y el Caribe (CEPAL). (2007). Cohesión social: inclusión y sentido de pertenencia en América Latina y el Caribe [LC/G.2335/REV.1]. Santiago de Chile: Naciones Unidas / CEPAL. https://repositorio.cepal.org/bitstream/ handle/11362/2812/2/S2007000_es.pdf 
Dempsey, N. (2009). Are good-quality environments socially cohesive? Measuring quality and cohesion in urban neighbourhoods. Town Planning Review, 80(3), 315-345. http:// doi.org/10.3828/tpr.80.3.5

Espinoza, V. \& Barozet, E. (2009). ¿De qué hablamos cuando decimos "clase media"? Perspectivas sobre el caso chileno. En A. Joignant \& P. Güell (Eds.), El arte de clasificar a los chilenos. Enfoques sobre los modelos de clasificación en Chile (pp. 103-130). Santiago, Chile: Ediciones Universidad Diego Portales.

Espinoza, V. \& Durston, J. (2013). Análisis de Redes Interculturales: capital social y mediación institucional. En J. Durston (Coord.), Pueblos Originarios y sociedad nacional en Chile: la interculturalidad en las prácticas sociales (pp. 274-288). Santiago, Chile: Programa de las Naciones Unidas para el Desarrollo (PNUD). http://www.onu.cl/onu/wp-content/ uploads/2013/10/Libro-Pueblos-Originarios-y-sociedad-nacional-en-Chile.pdf

García, C., Carrasco, J. \& Rojas, C. (2014). El contexto urbano y las interacciones sociales: dualidad del espacio de actividades de sectores de ingresos altos y bajos en Concepción, Chile. EURE, 4O(121), 75-90. http://dx.doi.org/10.4067/S025071612014000300004

Garretón, M. A. (1981). Modelo y proyecto político del régimen militar chileno. Documento de trabajo, FLACSO, (125). Publicado en Revista Mexicana de Sociología, 44(2) (1982), 355-372. http://dx.doi.org/10.2307/3540270

Granovetter, M. (1973). The strength of weak ties. American Journal of Sociology, 78(6), 13601380. https://doi.org/10.1086/225469

Hidalgo, R., Santana, L. \& Link, F. (2019). New neoliberal public housing policies: between centrality discourse and peripheralization practices in Santiago, Chile. Housing Studies, 34(3), 489-518. https://doi.org/10.1080/02673037.2018.1458287

Imilan, W. (2016). Políticas y luchas por la vivienda en Chile: el camino neoliberal. Working Papers Series. Contested Cities. http://repositorio.uchile.cl/handle/2250/141198

Kleit, R. \& Carnegie, N. B. (2011). Integrated or isolated? The impact of public housing redevelopment on social network homophily. Social Networks, 33(2), 152-165. https:// doi.org/10.1016/j.socnet.2011.01.001

Link, F., Mora, R., Greene, M. \& Figueroa, C. (2017). Patrones de sociabilidad en barrios vulnerables: dos casos en Santiago, Chile. Revista Bitácora Urbano Territorial, 27(3), 9-18. http://dx.doi.org/10.15446/bitacora.v27n3.42574

Link, F. \& Valenzuela, F. (2014). Sociabilidad en contextos de fragmentación urbana. Inclusión y exclusión metropolitana en Santiago de Chile. Proyección 17(8), 149-168. https:// bdigital.uncu.edu.ar/objetos_digitales/7530/09-proy-link.pdf

Link, F., Valenzuela, F. \& Fuentes, L. (2015). Segregación, estructura y composición social del territorio metropolitano en Santiago de Chile: Complejidades metodológicas en el análisis de la diferenciación social en el espacio. Revista de Geografía Norte Grande, (62), 151-168. http://dx.doi.org/10.4067/S0718-34022015000300009

Marques, E. (2010). ¿Cómo son las redes de los individuos en situación de pobreza en el Brasil urbano? Revista Hispana para el Análisis de Redes Sociales, 18(9), 219-251. http://doi. org $/ 10.5565 /$ rev/redes.394

Maturana, B. \& Horne, R. (2016). Towards socially integrated housing in Chile: assessing conviviality through two key housing projects. Open House International, 41(2), 6-14. http://repositorio.uchile.cl/handle/2250/140008 
Maturana, B., Vergara, L. \& Romano, S. (2016). Vivienda pública de mixtura social en la ciudad neoliberal: dinámicas de integración social en Villa Las Araucarias, La Serena, Chile. En Congreso Internacional Contested Cities: del conflicto urbano a la construcción de alternativas, Eje 2, Artículo n ${ }^{\circ} 2-516$. Madrid: Facultad de Derecho, Universidad Autónoma de Madrid, 3-4 julio 2016. http://repositorio.uchile.cl/ handle/2250/140007

Méndez, M. (2008). Middle class identities in a neoliberal age: tensions between contested authenticities. The Sociological Review, 56(2), 220-237. https://doi.org/10.1111/ j.1467-954X.2008.00785.x

Méndez, M. \& Gayo, M. (2018). Upper middle class social reproduction. Wealth, schooling, and residential choice in Chile. Cham, Suiza: Palgrave. https://doi.org/10.1007/978-3-31989695-3

Raman, S. (2010). Designing a liveable compact city: physical forms of city and social life in urban neighbourhoods. Built Environment, 36(1), 63-80. https://doi.org/10.2148/ benv.36.1.63

Rasse, A. (2015). Juntos pero no revueltos: procesos de integración social en fronteras residenciales entre hogares de distinto nivel socioeconómico. EURE, 41(122), 125143. http://dx.doi.org/10.4067/S0250-71612015000100006

Rincón, L. (2015). Redes personales y capital social en una parroquia rural de la Provincia de Maracaibo (Venezuela) a fines del siglo xviII: La Inmaculada Concepción de La Cañada (1784-1804). HISTOReLo, 7(14), 13-59. http://dx.doi.org/10.15446/ historelo.v7n14.46821

Rodríguez, A. \& Sugranyes, A. (2004). El problema de vivienda de los "con techo". EURE, 30(91), 53-65. http://dx.doi.org/10.4067/S0250-71612004009100004

Ruiz-Tagle, J. (2016). La persistencia de la segregación y la desigualdad en barrios socialmente diversos: un estudio de caso en La Florida, Santiago. EURE, 42(125), 81-108. http:// dx.doi.org/10.4067/S0250-71612016000100004

Ruiz-Tagle, J., Labbé, G., Rocco, V., Schuster, J. P. \& Muñoz, J. C. (2019). Recuperación de plusvalías para financiar la inserción de viviendas sociales en barrios consolidados. En I. Irarrázabal, E. Piña, M. Letelier \& M. I. Jeldes (Eds.), Propuestas para Chile: Concurso de Politicas Públicas 2018 (pp. 115-161). Santiago, Chile: Pontificia Universidad Católica de Chile.

Ruiz-Tagle, J. \& Romano, S. (2019). Mezcla social e integración urbana: aproximaciones teóricas y discusión del caso chileno. INVI, 34(95), 45-69. http://revistainvi.uchile.cl/ index.php/INVI/article/view/1442

Sabatini, F. (2000). Reforma de los mercados de suelo en Santiago, Chile: efectos sobre los precios de la tierra y la segregación residencial. EURE, 26(77), 49-80. http://dx.doi. org/10.4067/S0250-71612000007700003

Sabatini, F. \& Brain, I. (2008). La segregación, los guetos y la integración social urbana: mitos y claves. EURE, 34(103), 5-26. http://dx.doi.org/10.4067/S0250-7161200 8000300001

Sabatini, F., Brain, I. \& Prieto, J. (2011). Subsidio a la localización: desnaturalización de una política de integración social urbana. Curso sobre políticas e instrumentos de gestión de suelo. Universidad Nacional de Colombia (UnAL) y Lincoln Policy Institute, Medellín, agosto 22-24, 2011. 
Sabatini, F., Cáceres, G. \& Cerda, J. (2001). Segregación residencial en las principales ciudades chilenas: Tendencias de las tres últimas décadas y posibles cursos de acción. EURE, 27(82), 21-42. http://dx.doi.org/10.4067/S0250-71612001008200002

Sabatini, F., Mora, P., Polanco, I. \& Brain, I. (2014). Conciliando integración social y negocio inmobiliario: seguimiento de proyectos integrados (PIS) desarrollados por inmobiliarias e implicancias de política. En M. Smolka \& F. Furtado, Instrumentos notables de politicas de suelo en América Latina (pp. 61-69). Quito: Lincoln Institute of Land Policy.

Sabatini, F., Rasse, A., Mora, P. \& Brain, I. (2012). ¿Es posible la integración residencial en las ciudades chilenas?: Disposición de los grupos medios y altos a la integración con grupos de extracción popular. EURE, 38(115), 159-194. http://dx.doi.org/10.4067/ S0250-71612012000300008

Sabatini, F. \& Salcedo, R. (2007). Gated communities and the poor in Santiago, Chile: Functional and symbolic integration in a context of aggressive capitalist colonization of lower-class areas. Housing Policy Debate, 18(3), 577-606. https://doi.org/10.1080/ 10511482.2007.9521612

Sabatini, F. \& Vergara, L. (2018). ¿Apoyo a lugares o apoyo a personas? Dos proyectos chilenos de vivienda socialmente integrada. INVI, 33(94), 9-48. http://revistainvi.uchile.cl/ index.php/INVI/article/view/1337

Sampson, R. J., Morenoff, J. D. \& Gannon-Rowley, T. (2002). Assessing "neighborhood effects": social processes and new directions in research. Annual Review of Sociology, 28(1), 443-478. https://doi.org/10.1146/annurev.soc.28.110601.141114

Stillerman, J. (2016). Educar a niños y niñas de clase media en Santiago: capital cultural y segregación sociocultural en la formación de mercados locales de educación. EURE, 42(126), 169-186. http://dx.doi.org/10.4067/S0250-71612016000200008

Talen, E. (2002). The social goals of new urbanism. Housing Policy Debate, 13(1), 165-188. https://doi.org/10.1080/10511482.2002.9521438

Tironi, E. (2007). Cohesión social en Chile. El retorno de un viejo tema. Quórum. Revista de pensamiento iberoamericano, (18), 42-50. https://dialnet.unirioja.es/servlet/articulo?co digo $=2560255$

Torche, F. (2005). Unequal but fluid: social mobility in Chile in comparative perspective. American Sociological Review, 70(3), 422-450. https://doi.org/10.1177\% 2F000312240507000304

Vergara, L. (2019). Mixtura y cohesión social de barrio: una aproximación socio-espacial a las nuevas políticas de vivienda de Latinoamérica. Andamios, 16(40), 275-298. http:// dx.doi.org/10.29092/uacm.v16i40.707

Watt, P. (2009). Living in an oasis: middle-class disaffiliation and selective belonging in an English suburb. Environment and planning A, 41(12), 2874-2892. https://doi. org/10.1068\%2Fa41120

Webber, R. (2007). The metropolitan habitus: its manifestations, locations, and consumption profiles. Environment and Planning A, 39(1), 182-207. https://doi. org $/ 10.1068 \% 2 \mathrm{Fa} 38478$ 